\title{
An antibody present in everybody that attacks malaria infected erythrocytes
}

\author{
James Kennedy
}

Manatee Memorial Hospital, Bradenton, USA

Email: rkenned9@tampabay.rr.com

Received 13 May 2013; revised 26 May 2013; accepted 20 June 2013

Copyright (C) 2013 James Kennedy. This is an open access article distributed under the Creative Commons Attribution License, which permits unrestricted use, distribution, and reproduction in any medium, provided the original work is properly cited.

\begin{abstract}
These malaria targeting antibodies are band 3 antibodies and they recognize a special configuration of a molecule called band 3 that is present on erythrocytes. The special band 3 configuration is present on the surface of senescent erythrocytes, malaria infected erythrocytes, the erythrocytes of certain hemoglobinnopathies such as sickle cell disease and on the erythrocytes of some metabolic disorders such as G6PD. Note that these hemoglobinopathies and metabolic disorders all aid in the survival of falciparum malaria to such an extent that their incidence is increased in falciparum endemic areas [1-3]. Though there are many adhesive molecules involved in the pathology of falciparum malaria and sickle cell anemia, the focus here is on the band 3 molecules.
\end{abstract}

Keywords: Band 3; Malaria; Sickle

\section{INTRODUCTION}

This antibody not only targets erythrocytes that display the special band 3 configurations for reticuloendothelial elimination, it also prevents their endothelial adhesion $[1,4]$. These two actions have the potential to eliminate malaria infected erythrocytes before their parasites can mature and infect other erythrocytes and to reduce the pathologic microvascular occlusion. The question is, do the hemoglobinopathies and metabolic disorders whose erythrocytes display the band 3 configurations manifest their malaria surviving effect because of this? Any attempt to answer this requires an examination of the band 3 molecule and its involvement in these disorders.

\section{METHODS}

The band 3 molecules are dispersed on the surface of each erythrocyte where they normally function as bicar- bonate channels. As erythrocytes age their hemoglobin molecules begin to break down and form hemichromes that adhere to the cytoplasmic surface of the cell membrane and cause the surface band 3 molecules in the vicinity to cluster above them. This clustering exposes previously hidden adhesive antigenic band 3 peptides which are recognized by natural band 3 antibodies that will, in concert with a complement component, target senescent erythrocytes for reticuloendothelial elimination in the spleen and will also block their adhesion to the endothelium's band 3 receptor [4]. This mechanism for the physiologic removal of senescent erythrocytes after their normal life span appears to be the same one that removes cluster displaying malaria infected erythrocytes and is involved in the pathology that occurs in hemoglobinnopathies such as sickle cell disease.

Is there a correlation between the number of band 3 clusters displayed on erythrocytes and the band 3 antibody titer? Two different studies, one measuring band 3 clusters on erythrocytes in hemoglobin $\mathrm{C}$ anemia and the other measuring band 3 antibodies in sickle cell disease (SCD) suggest possible answers. The first study documents the frequency of band 3 clusters on falciparum infected and uninfected erythrocytes in hemoglobin (Hgb) C anemia, a hemoglobinopathy. Hgb AA erythrocytes were compared to erythrocytes containing Hgb CC and trait erythrocytes with Hgb AC. The cluster levels in uninfected erythrocytes were lowest in those with hemoglobin AA, higher in trait AC and highest in homozygous CC hemoglobin. Infected erythrocytes had higher cluster levels in all 3 hemoglobin types with the same order of progressive increases [5]. It is important to note that there are clusters present on Hgb AA erythrocytes where their antigenic recognition is responsible for the basil level of band 3 antibodies. It is significant that an inverse correlation exist between the cluster frequency and the occurrence of parasitemia in malaria endemic areas. A study in Kenya [6] measured the malaria parasite fre- 
quency in those with Hgb AA, AS and SS. Parasites were found in $35.8 \%$ with AA, $5.8 \%$ in AS and $0.5 \%$ SS. There were fewer subjects with Hgb SS in the study because those with SCD have a difficult time surviving a falciparum attack because of the serious nature of this disease, however, those survivors are much more resistant to recurrent infection. The fact that erythrocytes containing Hgb AA and Hgb AC have clusters on their surface but don't have anemia suggests that until a critical number of these molecules is reached, perhaps when their cloaking antibodies can cross link, anemia does not occur.

The other study measured band 3 antibody levels in a different hemoglobinopathy, sickle cell disease (SCD) [7]. The antibody levels were measured in steady state SCD, in sickle cell trait, in sickle cell crisis and in Hgb AA erythrocyte controls. Surprisingly, the antibody levels were slightly higher in Hgb AA (9.64) and Hgb AS (9.43) than in Hgb SS (8.36) but the level found during a sickle crisis (2.86) was almost $1 / 3$ that in the steady state SS SCD. The cluster count on erythrocytes with SS Hgb would logically be the highest of those tested, as it was in Hgb CC erythrocytes, so why wasn't the antibody level elevated?

In these two studies it appears that the band 3 antibody levels did not increase as would be expected as the cluster count increased. However, the antibody levels in the study were measured in the serum and did not account for those that cloaked band 3 clusters on erythrocytes and the antibodies being lost on erythrocytes removed in the spleen. As the cluster levels went up more antibodies were removed from the serum and when anemia developed in Hgb C anemia and SCD the antibody/cluster complexes was removed from the blood volume and the antibody level went down. Antibody production will require significant up regulation once anemia develops and in SCD it may not be able to fully cloak those erythrocytes displaying clusters and endothelial adhesion will begin. It is suggested that there is an insufficient amount of band 3 antibody to cloak all of the cluster bearing erythrocytes in SCD resulting in varying degrees of endothelial adhesion where extreme antibody insufficiency is present a crisis attack. This antibody insufficiency could occur due to excessive amounts of cluster bearing erythrocytes or decreased antibody production but the first seems more likely.

In the sickle trait antibody recognition of their increased cluster numbers may provide enough antigenic stimulation of the B cell clones responsible for the band 3 antibodies to provide a head start in fighting a falciparum infection. The obstruction of the microvascular flow that occurs in a sickle cell crisis is analogous to that condition in falciparum infections but an infection that occurs in patients with one of the mild forms of hemoglo- binopathies the antigenic stimulus for band 3 antibodies appears to be enough to reduce the severity of the infection.

What is the cluster trigger for erythrocyte removal and for their endothelial adhesion? The most likely trigger for anemia is for sufficient numbers of clusters to be present to allow cross linking of band 3 antibodies [8]. Except in rare cases the hemoglobin molecules in an erythrocyte do not break down all at once and the numbers of cluster/ antibody complexes on an erythrocyte will normally increase until a level is reached where antibody cross linking occurs. As far as a trigger for endothelial adhesion, any presence of adhesive band 3 clusters on erythrocytes should increase the transit time in the microvascular channels and significant increases could lead to a sickle crisis.

\section{DISCUSSION}

One experiment demonstrates a direct involvement of band 3 molecules in a falciparum infection, an injection of the adhesive band 3 peptides was given to falciparum infected monkeys and this resulted in the appearance of mature forms of intra-erythrocyte parasites in the circulation 24 hours after the injection [9]. The implication is that these peptides blocked the endothelial adhesion of band 3 clusters on infected erythrocytes preventing their endothelial sequestration and causing them to appear in the circulation where immunologic recognition of parasite antigens could occur.

\section{CONCLUSION}

The hypothesis presented is that the antigenic stimulation by band 3 clusters to produce antibodies increases as their numbers do but anemia does not occur until the number of clusters is such that their antibodies can cross the link. A mild anemia develops in homozygous hemoglobin $\mathrm{C}$ anemia without endothelial adhesion because antibodies are cloaking all the clusters but in the more severe sickle cell anemia antibody production cannot meet the demand for erythrocyte removal and the surplus of erythrocytes displaying uncloaked band 3 clusters begins to adhere to the vascular endothelium. In a sickle cell crisis certain conditions cause increased breakdown of the hemoglobin molecule resulting in larger numbers of cluster displaying uncloaked erythrocytes and endothelial adhesion increases to the point that microvascular obstruction occurs. The microvascular obstruction that occurs in a sickle crisis condition is analogous to that in a falciparum infection where the parasite infected erythrocytes also display more band 3 clusters on their surface than can be cloaked by antibodies. In both falciparum infections and sickle cell anemia there are multiple adhesive mechanisms in play but information presently avail- 
able suggests that it may be possible to influence the band 3 clusters participation in their pathological vasoocclusion. The sickle cell trait's ability to beneficially modify the course of a falciparum infection due to its presumed ability to stimulate band 3 antibodies production that can cloak the adhesive band 3 clusters present on the infected erythrocytes suggests the possibility of producing a vaccine with the adhesive band 3 peptides [9]. Such a vaccine would be particularly valuable when given in infancy where it would increase those children's chance of surviving a falciparum infection while developing immunity to the local parasite strain.

\section{REFERENCES}

[1] Crandall, I., Guthrie, N. and Sherman, I.W. (1995) Plasmodium falciparum: Sera of individuals living in a malaria-endemic region recognize peptide motifs of the human erythrocyte anion transport protein. The American Journal of Tropical Medicine and Hygiene, 52, 450-455.

[2] Ayi, K., Turrini, F., Piga, A. and Arese, P. (2004) Enhanced phagocytosis of ringparasitizedmutant erythrocytes: A common mechanism that mayexplain protection against falciparum malaria in sickle trait andbeta-thalassemia trait. Blood, 104, 3364-3371. doi:10.1182/blood-2003-11-3820

[3] Hogh, B., Petersen, E., Crandall, I., Gottschau, A. and Sherman, I.W. (1994) Immune responses to band 3 neoantigens on Plasmodium falciparum-infected erythrocytes in subjects living in an area of intense malaria transmission are associated with low parasite density and high hematocrit value. Infection and Immunity, 62, 43624366.
[4] Pantaleo, A., Giribaldi, G., Mannu, F., Arese, P. and Turrini, F. (2008) Naturally occurring anti-band 3 antibodies and red blood cell removal under physiological and pathological conditions. Autoimmunity Review, 7, 457462. doi:10.1016/j.autrev.2008.03.017

[5] Tokumasu, F., Fairhurst, R.M., Ostera, G.R., Brittain, N.J., Hwang, J., Wellems, T.E. and Dvorak, J.A. (2005) Band 3 modifications in Plasmodium falciparum-infected AA and CC erythrocytes assayed by autocorrelation analysis using quantum dots. Journal of Cell Science, 118, 1091-1098. doi:10.1242/jcs.01662

[6] Aluoch, J.R. (1997) Higher resistance to Plasmodium falciparum infection in patients with homozygous sickle cell disease in western Kenya. Tropical Medicine \& International Health, 2, 568-571. doi:10.1046/j.1365-3156.1997.d01-322.x

[7] Villaaescusa, R., Arce, A., Lelanne-Mistrih, M., Lamarre, Y., Hierso, R., Hernandez, C. and Hardy-Dessources, M. (2012) Natural antiband 3 antibodies in patients with sickle cell disease. Comptes Rendus Biologies. doi:10.1016/j.crvi.2012.09.002

[8] Naughton, B.A., Dornfest, B.S., Bush, M.E., Carlson, C.A. and Lapin, D.M. (1990) Immune activation is associated with phenylhydrazine-induced anemia in the rat. Journal of Laboratory and Clinical Medicine, 116, 498507.

[9] Crandall, I., Collins, W., Gysin, J. and Sherman, I.W. (1993) Synthetic peptides based on motifs present in human band 3 protein inhibit cytoadherence/sequestration of the malaria parasite Plasmodium falciparum. Proceedings of the National Academy of Sciences of the United States of America, 90, 4703-4707. doi:10.1073/pnas.90.10.4703 